\title{
Equivalent Bilinear Elastic Single Degree of Freedom System of Multi Degree of Freedom Structure with Negative Stiffness
}

\author{
Hong-Nan $\mathrm{Li}^{1}$, Chunxu $\mathrm{Qu}^{1, * \dagger}$, Linsheng Huo ${ }^{1}$ and Satish Nagarajaiah ${ }^{2}$ \\ 1. State Key Laboratory of Coastal and Offshore Engineering, Dalian University of Technology, \\ Dalian, China \\ 2. Department of Civil and Environmental Engineering, Rice University, Houston, USA
}

\begin{abstract}
The advantage of negative stiffness (NS) is to reduce the structural internal force, especially base shear, which is necessary to be considered during structural design. During design procedures, the structural response should be estimated. For the purpose of estimating the response of the structure with NS, this paper presents an innovative approximate method to transfer a multi degree of freedom (MDOF) structure with NS in the $1^{\text {st }}$ story (MNS1) to an approximate single degree of freedom (SDOF) bilinear elastic system (ASBS). The accuracy of approximation from the MNS1 to the ASBS is studied by the sensitivity method of mode shapes in which the relative changes in structural mode shapes are related to the variation of stiffness. An eight-story numerical example is employed to illustrate the accuracy of approximation by the proposed method. The results show that the accuracy of approach is acceptable, and ASBS can estimate the response of the MNS1.
\end{abstract}

KEY WORDS: response evaluation; modal superposition; approximation accuracy; equivalent SDOF system

\section{Introduction}

For the earthquake engineering, the most important function of structural stiffness is to resist ambient excitation and prevent structural inter-story deformation. Although the larger stiffness can resist the more external force, the inter-story shear force would be increased with the increasing of stiffness[1, 2]. The concept of stiffness weakening was proposed by Reinhorn et al. [3] and Viti et al.[4], in which the internal force or acceleration is attenuated by the reducing of structural stiffness. There is some research on the isolator with negative stiffness for the application of vehicles, whose negative stiffness induces the stiffness weakening $[5,6]$. However, if the structure is actually weakened by reducing the strength of beams (or in some cases beams and columns), it can result in early yielding and permanent deformation, which would be very dangerous for structural safety. Antoniadis et al.[7] proposed a novel vibration damping and isolation concept using negative stiffness, which did not decrease the

\footnotetext{
* Correspondence to: Chunxu Qu, Faculty of Infrastructure Engineering, Dalian University of Technology, Dalian 116024, China

† E-mail: qcx0311@163.com
} 
overall structural stiffness with embedded negative stiffness elements, and counteracted the inertial and excitation forces by the proper redistribution and reallocation of the stiffness and the damping elements of the system. Nagarajaiah et al.[8] proposed the "apparent weakening" that mimics the early "yielding" without inducing the structural inelastic behavior, and makes the structure remain elastic. The realization of the "apparent weakening" can be performed by the negative stiffness (NS), which can be generated by the negative stiffness device (NSD) [9-11].

In order to make sure the safety of a structure, the structural response should be estimated during structural design. Due to the advantage of NS to reduce the structural internal force, it is necessary to consider the NS property during estimating structural response. Although time-history analysis of an elaborate analytical model is probably one of the best options, its applicability requires large computational time and effort for establishing elaborate model and for the estimation of a structural response under seismic loading condition. In civil engineering, many building codes adopted equivalent single-degree-of-freedom (SDOF) systems to evaluate the overall performance of the structure, which do not need to perform the time-consuming computation for the elaborate analytical model. Most of multi-degree-of-freedom (MDOF) structures are always regarded to be linear elastic, which can be converted to SDOF system easily by mode superposition method [12]. However, a MDOF structure with NS that exhibits nonlinear property is hard to be transferred to an equivalent SDOF system. There are several studies on this conversion for the nonlinear system. Chopra and Goel [13] proposed the mode pushover analysis (MPA) method which decomposes the structure according to the mode superposition, and use the static pushover to determine the yielding point for each mode. They also pointed out that the MPA lacks a rigorous theory but it is simple and effective in most cases for structural designs. Makarios [14] defined an equivalent nonlinear SDOF system to represent the MDOF structure through the pushover procedure. Tjhin et al.[15] presented an energy-based pushover approach, which established a more accurate capacity curve to provide a better estimation of the peak roof displacement. Vamvatsikos and Cornell [16] exploited the static pushover and incremental dynamic analysis to obtain an accurate estimate. Pasala et al. [17] investigated the SDOF building with the NSD and its linear or bilinear elastic behavior. Ray et al. [18] studied the nonlinear inelastic behavior of the NSD in the SDOF system. Pasala et al. [19] have studied MDOF with NSD in first floor. Yang et al. [20] studied the dynamic and power flow behavior of a nonlinear vibration isolation system with the negative stiffness mechanism, which can enlarge the frequency band for the effective vibration isolation. Zou and Nagarajaiah [21] investigated the piecewise linear dynamic oscillator with negative stiffness followed by positive stiffness, which derived approximate the periodic solutions. The conversion from the MDOF system with the NS to the SDOF, which is required for the design of real engineering structures, has not currently been studied with related research.

This paper presents a new approximate method to convert the bilinear elastic MDOF to an equivalent SDOF. The MDOF structure with the NS only in the $1^{\text {st }}$ story (MNS1) is considered, which is quite effective in reducing the base shear and displacement. Based on the mode superposition method [12], the conversion is derived to obtain the approximate SDOF bilinear elastic system 
(ASBS). The accuracy of approximation from the MNS1 to the ASBS is analyzed by the sensitivity method for mode shapes proposed by Zhao and DeWolf [22] who investigated the relative changes in the structural mode shape due to the changes in the structural stiffness. An eight-story numerical example is employed to illustrate the accuracy of approximation by the proposed method. Different cases, such as different ground motions, different NS parameters, and the structures with different natural frequencies, are considered to exhibit the effectiveness of the proposed method.

It should be noted that the NS could increase the structural deformation in the floor and result in the inelastic behavior of structure, which can be prevented by the supplemental damping. However, the study in this paper is the basic research for the negative stiffness, which only focuses on the elastic behavior without the supplemental damping.

\section{Model definition}

Different from the restoring force provided by the positive structural stiffness against the deformation, the negative stiffness "restoring force" assists deformation. The original stiffness and ideal negative stiffness are shown in Fig. 1(a) and (b).

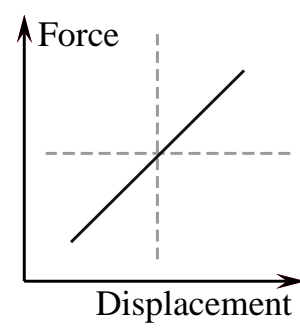

(a)

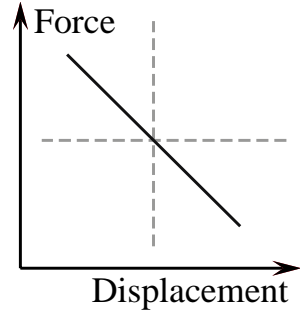

(b)

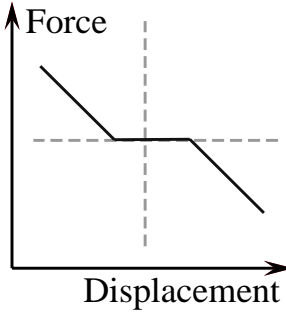

(c)

Fig. 1. Schematic diagrams depicting the force-deformation behavior: (a) positive stiffness; (b) negative stiffness; (c) negative stiffness with gap.

The negative stiffness can weaken the structure when added to the original structural stiffness. However, it would be helpful to guarantee the stability of structures to maintain the original stiffness under some small excitation [17]. Thus, the practical negative stiffness will have the force-deformation relation as in Fig. 1(c). The combination of the original stiffness and the negative stiffness in Fig. 1(c) is shown in Fig. 2.

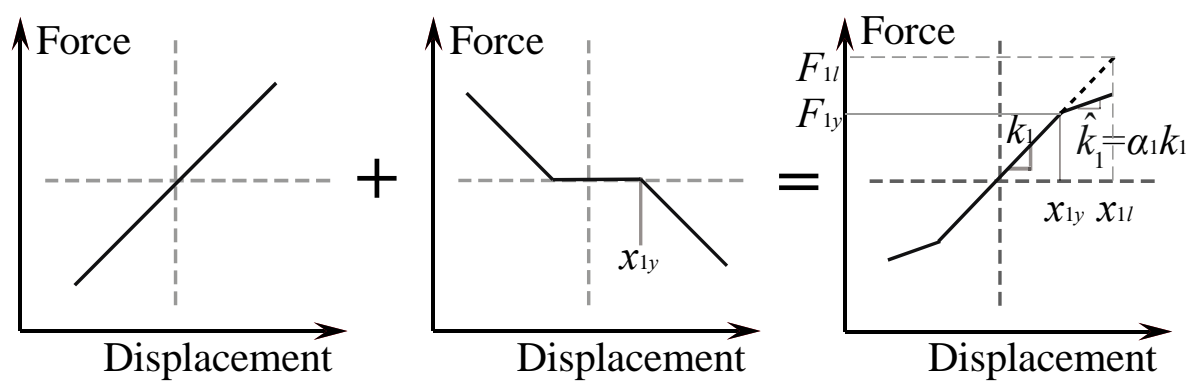

Fig. 2. Combination of the positive stiffness and the negative stiffness with gap results in bilinear 
stiffness.

Considering an $n$ degree-of-freedom shear-frame structure, the dynamics can be described by a second-order differential equation as follows

$$
\mathbf{M} \ddot{\mathbf{x}}+\mathbf{C} \dot{\mathbf{x}}+\mathbf{K x}=-\mathbf{M l} \ddot{x}_{g}
$$

where $\ddot{x}_{g}$ is the earthquake ground acceleration; $\mathbf{l} \in \mathfrak{R}^{n \times 1}$ is the vector whose element value is equal to $1 ; \mathbf{M}, \mathbf{C}, \mathbf{K} \in \mathfrak{R}^{n \times n}$ are the mass, damping, and stiffness constant matrices, respectively, which satisfy the following matrix architecture,

$\mathbf{M}=\left[\begin{array}{cccc}m_{1} & 0 & \cdots & 0 \\ 0 & m_{2} & \ddots & \vdots \\ \vdots & \ddots & \ddots & 0 \\ 0 & \cdots & 0 & m_{n}\end{array}\right], \mathbf{C}=\left[\begin{array}{cccc}c_{1}+c_{2} & -c_{2} & 0 & 0 \\ -c_{2} & c_{2}+c_{3} & \ddots & 0 \\ 0 & \ddots & \ddots & -c_{n} \\ 0 & 0 & -c_{n} & c_{n}\end{array}\right], \mathbf{K}=\left[\begin{array}{cccc}k_{1}+k_{2} & -k_{2} & 0 & 0 \\ -k_{2} & k_{2}+k_{3} & \ddots & 0 \\ 0 & \ddots & \ddots & -k_{n} \\ 0 & 0 & -k_{n} & k_{n}\end{array}\right]$

where $m_{i}, c_{i}$, and $k_{i}$ are the $i$-th story mass, damping and stiffness, respectively. This paper focuses on the MDOF shear-frame structure described in Eq.(1) with the additional negative stiffness property in the $1^{\text {st }}$ story (MNS1) as shown in Fig. 3(a). The force-deformation behavior in each story is described as Fig. 3(b).

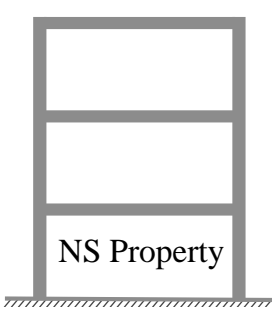

(a)

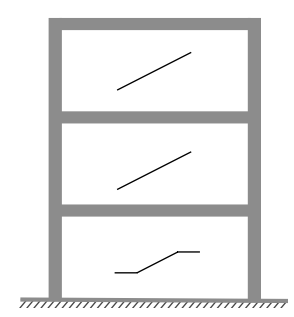

(b)

Fig. 3. Schematic diagrams depicting MNS1: (a) MNS1 structure; (b) force-deformation behavior in each story of MNS1 structure

The dynamics of the MNS1 subjected to the earthquake ground motion is,

$$
m_{1} \ddot{x}_{1}+c_{1} \dot{x}_{1}+F_{1}\left(R_{1 y}, \alpha_{1}, x_{1}\right)=-m_{1} \ddot{x}_{g}
$$

where the value of the restoring force $F_{1}$ is decided by the parameters $R_{1 y}, \alpha_{1}$ and $x_{1}$, which are the "yielding" strength reduction factor, stiffness degradation, and the displacement for the $1^{\text {st }}$ floor, respectively. These parameters as shown in Fig. 2 can be expressed by 


$$
\begin{gathered}
R_{1 y}=\frac{F_{1 l}}{F_{1 y}}=\frac{x_{1 l}}{x_{1 y}} \\
\alpha_{1}=\frac{\hat{k}_{1}}{k_{1}}
\end{gathered}
$$

where $x_{1 l}\left(\right.$ or $\left.F_{1 l}\right)$ is the peak value of the $1^{\text {st }}$ floor deformation (or restoring force) for the corresponding linear system in Eq.(3) with $F_{1}=k_{1} x_{1}, x_{1 y}$ (or $F_{1 y}$ ) is the first floor "yielding" deformation ("yielding" restoring force) and $\hat{k}_{1}$ is the stiffness of structure after "yielding". So the restoring force $F_{1}$ of the first floor can be given by

$$
F_{1}\left(R_{1 y}, k_{1 d}, x_{1}\right)=\left\{\begin{array}{c}
k_{1} x_{1}, \text { if } x_{1} \leq x_{1 y} \\
k_{1} x_{1 y}+\hat{k}_{1}\left(x_{1}-x_{1 y}\right), \text { if } x_{1}>x_{1 y}
\end{array}\right.
$$

Considering the dynamic behavior of the first floor in Eq.(3) and linear elastic structure in Eq.(1), the dynamics of MNS1 is described as,

$$
\mathbf{M} \ddot{\mathbf{x}}+\mathbf{C} \dot{\mathbf{x}}+\mathbf{F}\left(R_{1 y}, \alpha_{1}, x_{1}\right)=-\mathbf{M l} \ddot{x}_{g}
$$

where $\mathbf{M}, \mathbf{C} \in \mathfrak{R}^{n \times n}$ are the mass, damping matrices of the MNS1, respectively; the relative displacement vector $\mathbf{x} \in \mathfrak{R}^{n \times 1}$ is defined by:

$$
\mathbf{x}=\left(\begin{array}{llll}
x_{1} & x_{2} & \ldots & x_{n}
\end{array}\right)^{\mathrm{T}}
$$

$\mathbf{F}$ in Eq.(7) is the restoring force vector, whose value is decided by the $1^{\text {st }}$ floor parameters $R_{1 y}, \alpha_{1}$ and $x_{1}$, and expressed in a piecewise formulation

$$
\mathbf{F}\left(R_{1 y}, \alpha_{1}, x_{1}\right)=\left\{\begin{array}{c}
\mathbf{K} \mathbf{x}, \text { if } x_{1} \leq x_{1 y} \\
\mathbf{K} \mathbf{x}_{y}+\hat{\mathbf{K}}\left(\mathbf{x}-\mathbf{x}_{y}\right), \text { if } x_{1}>x_{1 y}
\end{array}\right.
$$

where $\mathbf{x}_{y}$ and $\hat{\mathbf{K}}$ represents the structural relative displacement vector and the stiffness matrix when $x_{1}$ reaches the "yielding" displacement $x_{1 y}, \hat{\mathbf{K}}$ has the matrix architecture as,

$$
\hat{\mathbf{K}}=\left[\begin{array}{cccc}
\hat{k}_{1}+k_{2} & -k_{2} & 0 & 0 \\
-k_{2} & k_{2}+k_{3} & \ddots & 0 \\
0 & \ddots & \ddots & -k_{n} \\
0 & 0 & -k_{n} & k_{n}
\end{array}\right]
$$

The following assumptions are valid in this study: (i) the word "yielding" in the model will not result in the non-restorable plastic deformation, which exhibits only a bilinear elastic behavior (no inelastic behavior); (ii) the structural plastic behavior is not considered during the deformation in each 
story here, and the shear-frame structure maintains the elastic stiffness that contains bilinear elastic behavior in the $1^{\text {st }}$ story and linear elastic in the other stories.

\section{Conversion from MNS1 to ASBS}

For a bilinear elastic structure, such as the MNS1 mentioned above, it is necessary to convert the MNS1 to a SDOF system. This section will derive this transformation from the MN1S to an approximate SDOF bilinear elastic system (ASBS).

For the linear elastic structure with the matrix parameters $\mathbf{M}, \mathbf{C}$ and $\mathbf{K}$ in Eq.(2), the natural frequencies and mode shapes can be expressed as $\omega_{1}, \omega_{2}, \ldots, \omega_{\mathrm{n}}$, and $\boldsymbol{\Phi}_{1}, \boldsymbol{\Phi}_{2}, \ldots, \boldsymbol{\Phi}_{n}$. The co-ordinate $q_{1}, q_{2}, \ldots, q_{n}$ are governed by

$$
\ddot{q}_{i}+2 \zeta_{i} \omega_{i} \dot{q}_{i}+\omega_{i}^{2} q_{i}=-\Gamma_{i} \ddot{x}_{g}(t)
$$

where $\zeta_{i}$ is the damping ratio for the $i$-th mode and $\Gamma_{i}$ is expressed by

$$
\begin{gathered}
\Gamma_{i}=\frac{\boldsymbol{\Phi}_{i}^{\mathrm{T}} \mathbf{M l}}{M_{i}} \\
M_{i}=\boldsymbol{\Phi}_{i}^{\mathrm{T}} \mathbf{M} \boldsymbol{\Phi}_{i}
\end{gathered}
$$

For the structure with the stiffness $\hat{\mathbf{K}}$ in Eq.(10), the natural frequencies and mode shapes will be denoted as $\hat{\omega}_{1}, \hat{\omega}_{2}, \ldots, \hat{\omega}_{n}$, and $\boldsymbol{\Psi}_{1}, \boldsymbol{\Psi}_{2}, \ldots, \boldsymbol{\Psi}_{n}$. The co-ordinates are denoted as $p_{1}, p_{2}, \ldots, p_{n}$. Thus, in the bilinear elastic structure in Eq.(7), the mode expansion of displacement $\mathbf{x}$ in Eq.(8) has the form as the following,

$$
\mathbf{x}=\sum_{i=1}^{n}\left(\boldsymbol{\Phi}_{i} q_{i}+\boldsymbol{\Psi}_{i} p_{i}\right)=\boldsymbol{\Phi}_{1} q_{1}+\boldsymbol{\Phi}_{2} q_{2}+\ldots+\boldsymbol{\Phi}_{n} q_{n}+\boldsymbol{\Psi}_{1} p_{1}+\boldsymbol{\Psi}_{2} p_{2}+\ldots+\boldsymbol{\Psi}_{n} p_{n}
$$

Eq.(14) means that the co-ordinate $p_{i}$ is equal to zero when structure vibrates in linear part, and otherwise nonzero when vibrates in the bilinear part. As the derivation of Eq.(11) based on the superposition [12], substituting Eq.(14) into Eq.(7), pre-multiplying by $\boldsymbol{\Phi}_{i}$, and then is dividing both sides by $M_{i}$ gives,

$$
\ddot{q}_{i}+\Xi_{m}+2 \zeta_{i} \omega_{i} \dot{q}_{i}+\Xi_{c}+\omega_{i}^{2} q_{i}+\Xi_{k}=-\Gamma_{i} \ddot{x}_{g}
$$

where

$$
\begin{gathered}
\Xi_{m}=\frac{\boldsymbol{\Phi}_{i}^{\mathrm{T}} \mathbf{M} \boldsymbol{\Psi}_{1}}{M_{i}} \ddot{p}_{1}+\ldots+\frac{\boldsymbol{\Phi}_{i}^{\mathrm{T}} \mathbf{M} \boldsymbol{\Psi}_{j}}{M_{i}} \ddot{p}_{j}+\ldots+\frac{\boldsymbol{\Phi}_{i}^{\mathrm{T}} \mathbf{M} \boldsymbol{\Psi}_{n}}{M_{i}} \ddot{p}_{n} \\
\Xi_{c}=\frac{\boldsymbol{\Phi}_{i}^{\mathrm{T}} \mathbf{C} \boldsymbol{\Psi}_{1}}{M_{i}} \dot{p}_{1}+\ldots+\frac{\boldsymbol{\Phi}_{i}^{\mathrm{T}} \mathbf{C} \boldsymbol{\Psi}_{j}}{M_{i}} \dot{p}_{j}+\ldots+\frac{\boldsymbol{\Phi}_{i}^{\mathrm{T}} \mathbf{C} \boldsymbol{\Psi}_{n}}{M_{i}} \dot{p}_{n}
\end{gathered}
$$




$$
\Xi_{k}=\frac{\boldsymbol{\Phi}_{i}^{\mathrm{T}} \hat{\mathbf{K}} \boldsymbol{\Psi}_{1}}{M_{i}} p_{1}+\ldots+\frac{\boldsymbol{\Phi}_{i}^{\mathrm{T}} \hat{\mathbf{K}} \boldsymbol{\Psi}_{j}}{M_{i}} p_{j}+\ldots+\frac{\boldsymbol{\Phi}_{i}^{\mathrm{T}} \hat{\mathbf{K}} \boldsymbol{\Psi}_{n}}{M_{i}} p_{n}
$$

The coupling phenomenon of co-ordinates $q_{i}$ and $p_{i}$ is hidden in Eqs.(16) (18).According to the expression Eq.(3), neglecting the coupling co-ordinates gives the controlling equation of ASBS as,

$$
\ddot{\tilde{q}}_{i}+2 \zeta_{i} \omega_{i} \dot{\tilde{q}}_{i}+f_{i}\left(r_{i y}, \lambda_{i d}, \tilde{q}_{i}\right)=-\Gamma_{i} \ddot{x}_{g}
$$

where the new co-ordinate $\tilde{q}_{i}$ for the bilinear elastic system is equal to $p_{i}+q_{i}$; the factors of $r_{1 y}, \lambda_{1 d}$ like the parameters $R_{1 y}, \alpha_{1}$ defined in Eq.(4) and Eq.(5) which are the "yielding" strength reduction factor, frequency degradation factor for the ASBS, respectively, and can be expressed by

$$
\begin{gathered}
r_{i y}=\frac{q_{i l}}{q_{i y}}=\frac{f_{i l}}{f_{i y}} \\
\lambda_{i d}=\frac{\hat{\omega}_{i}^{2}}{\omega_{i}^{2}}
\end{gathered}
$$

where $q_{i l}$ (or $f_{i l}$ ) is the maximal co-ordinate value (or restoring force) of the linear elastic system in Eq.(11); $q_{i y}$ (or $f_{i y}$ ) is the "yielding" co-ordinate value (or restoring force), which has the following relation with $x_{1 y}$ in Eq.(4),

$$
\phi_{1 i} q_{i y}=x_{1 y}
$$

where $\phi_{1 i}$ is the $1^{\text {st }}$ element of $\boldsymbol{\Phi}_{i}$. So the restoring force $f_{i}$ in Eq.(19) can be expressed by

$$
f_{i}\left(r_{i y}, \lambda_{i d}, \tilde{q}_{i}\right)=\left\{\begin{array}{c}
\omega_{i}^{2} \tilde{q}_{i}, \text { if } \tilde{q}_{i} \leq q_{i y} \\
\omega_{i}^{2} q_{i y}+\hat{\omega}_{i}^{2}\left(\tilde{q}_{i}-q_{i y}\right), \text { if } \tilde{q}_{i}>q_{i y}
\end{array}\right.
$$

If the ASBS is used to estimate the MNS1 response, it is necessary to illustrate the precision of the approximation.

\section{Precision of approximate method}

According to Eq.(11) and Eq.(19), the difference is caused by the changes of natural frequencies and mode shapes. On the other hand, the bilinear elastic behavior of the MNS1 is caused by the $1^{\text {st }}$ floor stiffness changes, which means that $k_{1}$ is changed to $\hat{k}_{1}$ by the negative stiffness. So the precision of the approximation will be discussed according to the sensitivity of mode shapes with respect to the changing $1^{\text {st }}$ floor stiffness. Here, the sensitivity study proposed by Zhao and DeWolf [22] is employed, and it is used for the conversion from the MNS1 to the ASBS.

Note that $\mathbf{K}$ in Eq.(2) can also be expressed by 


$$
\mathbf{K}=\left[\begin{array}{cccc}
\alpha_{1} k_{1}+k_{2} & -k_{2} & 0 & 0 \\
-k_{2} & k_{2}+k_{3} & \ddots & 0 \\
0 & \ddots & \ddots & -k_{n} \\
0 & 0 & -k_{n} & k_{n}
\end{array}\right] \text {, when } \alpha_{1}=1
$$

The advantage of the above expression can be used to investigate the sensitivity in terms of stiffness degradation factor $\alpha_{1}$. According to the reference [22], the sensitivity coefficients for the mode shapes can be expressed by the combination of the mode shapes as follows,

$$
\frac{\partial \boldsymbol{\Phi}_{j}}{\partial \alpha_{1}}=\sum_{s=1}^{n} \beta_{s} \boldsymbol{\Phi}_{s}
$$

where

$$
\beta_{s}=\left\{\begin{array}{c}
\frac{k_{1}}{\left(\omega_{j}^{2}-\omega_{s}^{2}\right) M_{s}} \phi_{1 s} \phi_{1 j}, \text { if } j \neq s \\
0, \text { if } j=s
\end{array}\right.
$$

where $\phi_{1 j}$ is the $1^{\text {st }}$ element of $\boldsymbol{\Phi}_{j}$. The changed mode shape can be expressed by

$$
\boldsymbol{\Psi}_{j}=\boldsymbol{\Phi}_{j}+\left(\alpha_{1}-1\right) \sum_{s=1}^{n} \beta_{s} \boldsymbol{\Phi}_{s}
$$

Due to the additional negative stiffness on the $1^{\text {st }}$ floor, $k_{1}$ is changed, and the stiffness matrix is also changed from Eq.(2) to Eq.(10) or Eq.(24). The approximation accuracy should be illustrated according to two possibilities,

(1) When $i=j$ in Eqs.(16) (17), substituting Eq.(26) and Eq.(27) into $\boldsymbol{\Phi}_{i}{ }^{\mathrm{T}} \mathbf{M} \boldsymbol{\Psi}_{i}$ and $\boldsymbol{\Phi}_{i}{ }^{\mathrm{T}} \mathbf{C} \boldsymbol{\Psi}_{i}$, there are the following results,

$$
\begin{gathered}
\frac{\boldsymbol{\Phi}_{i}^{\mathrm{T}} \mathbf{M} \Psi_{j}}{M_{i}}=\frac{\boldsymbol{\Phi}_{i}^{\mathrm{T}} \mathbf{M}\left(\boldsymbol{\Phi}_{i}+\mathbf{0}\right)}{M_{i}}=1 \\
\frac{\boldsymbol{\Phi}_{i}^{\mathrm{T}} \mathbf{C} \boldsymbol{\Psi}_{j}}{M_{i}}=\frac{\boldsymbol{\Phi}_{i}^{\mathrm{T}} \mathbf{C} \boldsymbol{\Phi}_{i}}{M_{i}}=2 \zeta_{i} \omega_{i}
\end{gathered}
$$

Due to the element in Eq.(18), $\boldsymbol{\Phi}_{i}^{\mathrm{T}} \hat{\mathbf{K}} \boldsymbol{\Psi}_{j}=\boldsymbol{\Psi}_{j}^{\mathrm{T}} \hat{\mathbf{K}} \boldsymbol{\Phi}_{i}$, a similar simplification for the sensitivity coefficient of $\boldsymbol{\Phi}_{j}$ can be performed to compute the sensitivity coefficient of $\boldsymbol{\Psi}_{j}$. According to Eq.(25), the sensitivity coefficients for mode shape $\boldsymbol{\Psi}_{j}$ can be obtained as,

$$
\frac{\partial \boldsymbol{\Psi}_{j}}{\partial \alpha_{1}}=\sum_{s=1}^{n} \gamma_{s} \boldsymbol{\Psi}_{s}
$$

Similar results as Eq.(26) and Eq.(27) are, 


$$
\begin{gathered}
\gamma_{s}=\left\{\begin{array}{c}
\frac{k_{1}}{\left(\omega_{j}^{2}-\omega_{s}^{2}\right) M_{s}} \psi_{1 s} \psi_{1 j}, \text { if } j \neq s \\
0, \text { if } j=s
\end{array}\right. \\
\boldsymbol{\Phi}_{j}=\boldsymbol{\Psi}_{j}+\left(1-\alpha_{1}\right) \sum_{s=1}^{n} \gamma_{s} \boldsymbol{\Psi}_{s}
\end{gathered}
$$

where $\psi_{1 j}$ is the $1^{\text {st }}$ element of $\boldsymbol{\Psi}_{j}$. Considering $\mathbf{M}_{i}=\boldsymbol{\Phi}_{i}{ }^{\mathrm{T}} \mathbf{M} \boldsymbol{\Phi}_{i}=\boldsymbol{\Psi}_{i}^{\mathrm{T}} \mathbf{M} \boldsymbol{\Psi}_{i}$, transposing $\boldsymbol{\Phi}_{i}^{\mathrm{T}} \hat{\mathbf{K}} \boldsymbol{\Psi}_{j}$ to $\boldsymbol{\Psi}_{i}^{\mathrm{T}} \hat{\mathbf{K}} \boldsymbol{\Phi}_{j}$ and substituting Eq.(32) into $\boldsymbol{\Psi}_{i}^{\mathrm{T}} \hat{\mathbf{K}} \boldsymbol{\Phi}_{j}$ gives,

$$
\frac{\boldsymbol{\Phi}_{i}^{\mathrm{T}} \hat{\mathbf{K}} \boldsymbol{\Psi}_{i}}{M_{i}}=\frac{\boldsymbol{\Psi}_{i}^{\mathrm{T}} \hat{\mathbf{K}} \boldsymbol{\Psi}_{i}}{M_{i}}=\hat{\omega}_{i}^{2}
$$

(2) when $i \neq j$, substituting Eq.(26) and Eq.(27) into $\boldsymbol{\Phi}_{i}^{\mathrm{T}} \mathbf{M} \boldsymbol{\Psi}_{j}$ and $\boldsymbol{\Phi}_{i}^{\mathrm{T}} \mathbf{C} \boldsymbol{\Psi}_{j}$ gives,

$$
\begin{gathered}
\frac{\boldsymbol{\Phi}_{i}^{\mathrm{T}} \mathbf{M} \Psi_{j}}{M_{i}}=\left(\alpha_{1}-1\right) \frac{k_{1}}{\left(\omega_{j}^{2}-\omega_{i}^{2}\right) M_{i}} \phi_{1 i} \phi_{1 j} \\
\frac{\boldsymbol{\Phi}_{i}^{\mathrm{T}} \mathbf{C} \boldsymbol{\Psi}_{j}}{M_{i}}=2 \zeta_{i} \omega_{i}\left(\alpha_{1}-1\right) \frac{k_{1}}{\left(\omega_{j}^{2}-\omega_{i}^{2}\right) M_{i}} \phi_{1 i} \phi_{1 j}
\end{gathered}
$$

Substituting Eq.(31) and Eq.(32) into $\boldsymbol{\Phi}_{i}^{\mathrm{T}} \hat{\mathbf{K}} \boldsymbol{\Psi}_{i}$ yields,

$$
\frac{\boldsymbol{\Phi}_{i}^{\mathrm{T}} \hat{\mathbf{K}} \Psi_{j}}{M_{i}}=\hat{\omega}_{i}^{2}\left(1-\alpha_{1}\right) \frac{k_{1}}{\left(\omega_{j}^{2}-\omega_{i}^{2}\right) M_{i}} \psi_{1 i} \psi_{1 j}
$$

For Eq.(34), the value of part $k_{1} /\left(\omega_{j}^{2}-\omega_{i}^{2}\right) / M_{i}$ is close to 1 . However, the part $\left(\alpha_{1}-1\right) \phi_{1 i} \phi_{1 j}$ is always too small such that the whole value of the part $\left(\alpha_{1}-1\right) k_{1} \phi_{1 i} \phi_{1 j} /\left(\omega_{j}^{2}-\omega_{i}^{2}\right) / M_{i}$ is much smaller than 1 . So the actual value of $\Xi_{m}$ in Eq.(16) can be approximated by $\boldsymbol{\Phi}_{i}{ }^{\mathrm{T}} \mathbf{M} \boldsymbol{\Phi}_{i} / M_{i}$ as follows

$$
\Xi_{m} \approx \frac{\boldsymbol{\Phi}_{i}^{\mathrm{T}} \mathbf{M} \Psi_{i}}{M_{i}} \ddot{p}_{i}=\ddot{p}_{i}
$$

$\Xi_{c}$ in Eq.(17) and $\Xi_{k}$ in Eq.(18) can also be approximated in the similar way, respectively,

$$
\begin{gathered}
\Xi_{c} \approx \frac{\boldsymbol{\Phi}_{i}^{\mathrm{T}} \mathbf{C} \boldsymbol{\Psi}_{i}}{M_{i}} \dot{p}_{i}=2 \zeta_{i} \omega_{i} \dot{p}_{i} \\
\Xi_{k} \approx \frac{\boldsymbol{\Phi}_{i}^{\mathrm{T}} \hat{\mathbf{K}} \boldsymbol{\Psi}_{i}}{M_{i}} p_{i}=\hat{\omega}_{i}^{2} p_{i}
\end{gathered}
$$

Substituting Eqs.(37) (39) into Eq.(15), the SBS Eq.(15) can be approximated as,

$$
\ddot{q}_{i}+\ddot{p}_{i}+2 \zeta_{i} \omega_{i} \dot{q}_{i}+2 \zeta_{i} \omega_{i} \dot{p}_{i}+\omega_{i}^{2} q_{i}+\hat{\omega}_{i}^{2} p_{i}=-\Gamma_{i} \ddot{x}_{g}
$$

By setting $\tilde{q}_{i}=p_{i}+q_{i}$, Eq.(40) turns to be Eq.(19). 
The application procedures can be summarized as follows,

Step 1. For the linear part, convert Eq.(1) to Eq.(11) by the mode superposition. Calculate the parameters $\omega_{i}, \boldsymbol{\Phi}_{i}, \zeta_{i}$ and $\Gamma_{i}$.

Step 2. For the "yielding" part, replace the stiffness matrix $\mathbf{K}$ in Eq.(1) with $\hat{\mathbf{K}}$. As the Step 1, calculate the parameters $\hat{\omega}_{i}$ and $\boldsymbol{\Psi}_{i}$.

Step 3. According to the bilinear property as shown in Fig. 2 or in Eq.(20) (21), calculate the response $\tilde{q}_{i}$ of the SDOF bilinear system Eq.(19).

Step 4. Estimate the structural response under different modes including the displacement and base shear according to the following equations:

$$
\begin{gathered}
\tilde{x}_{j i}=\left\{\begin{array}{c}
\tilde{q}_{i} \Phi_{j i}, \text { if } \tilde{q}_{i} \leq q_{i y} \\
\left(\tilde{q}_{i}-q_{i y}\right) \Psi_{j i}+q_{i y} \Phi_{j i}, \text { if } \tilde{q}_{i}>q_{i y}
\end{array}\right. \\
\tilde{V}_{i}=\left\{\begin{array}{c}
\mathbf{E}^{\mathrm{T}} \mathbf{K} \boldsymbol{\Phi}_{i} \tilde{q}_{i}, \text { if } \tilde{q}_{i} \leq q_{i y} \\
\mathbf{E}^{\mathrm{T}} \hat{\mathbf{K}} \boldsymbol{\Psi}_{i}\left(\tilde{q}_{i}-q_{i y}\right)+\mathbf{E}^{\mathrm{T}} \mathbf{K} \boldsymbol{\Phi}_{i} q_{i y}, \text { if } \tilde{q}_{i}>q_{i y}
\end{array}\right.
\end{gathered}
$$

where $i$ represents the $i$-th mode; $j$ is the $j$-th floor; $\tilde{x}_{j i}$ and $\tilde{V}_{i}$ are the estimated $j$-th floor displacement and base shear under $i$-th mode, respectively.

Step 5. Estimate the structural overall response by summing the responses under different modes:

$$
\begin{aligned}
& \tilde{x}_{j}=\sum_{i=1}^{n} \tilde{x}_{j i} \\
& \tilde{V}=\sum_{i=1}^{n} \tilde{V}_{i}
\end{aligned}
$$

where $\tilde{x}_{j}$ and $\tilde{V}$ are the estimated floor displacement and base shear, respectively.

\section{Numerical validation}

\subsection{Structural example}

In order to illustrate the accuracy for the approximations, Eqs.(37) (39), an eight-story structure example is employed [23], which is simplified to shear-type and linear elastic structure. The mass for each floor is $2.7 \times 10^{5} \mathrm{~kg}$, and the elastic module for each story is $19.2 \times 10^{8} \mathrm{~N} \cdot \mathrm{m}^{2}$. The damping ratio for this structure is assumed to be $5 \%$. This paper considers that the additional negative stiffness property only exists in the $1^{\text {st }}$ story, and other stories maintain the linear elastic property. For the linear structure without the NS in the $1^{\text {st }}$ story, the mode parameters are shown in Fig. 4, 


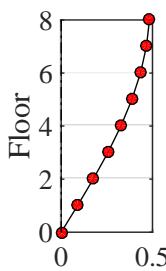

Shape

$T_{1}=0.4038 \mathrm{~s}$

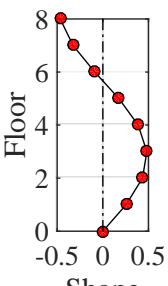

Shape

$T_{2}=0.1361 \mathrm{~s}$

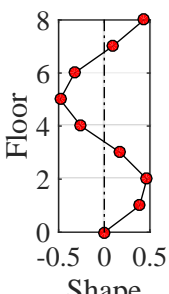

$T_{3}=0.0836$

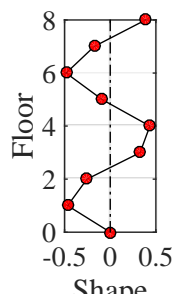$$
T_{4}=0.0618 \mathrm{~s}
$$

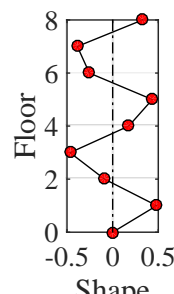

$T_{5}=0.0504 \mathrm{~s}$

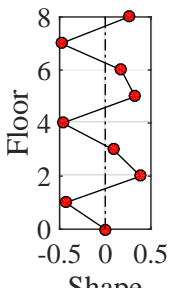$$
T_{6}=0.0438 \mathrm{~s}
$$
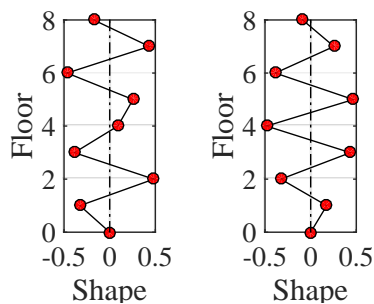

$T_{7}=0.0400 \mathrm{~s}$

$T_{8}=0.0379 \mathrm{~s}$

Fig. 4. Eight modes of the 8-storey structure.

Time histories of structural response during the earthquake are computed based on the classical Newmark integration and the classical modified Newton-Raphson method [12]. The calculation codes are complied and performed on MATLAB software platform (MathWorks, Natick, MA, USA).

The excitation is chosen as the earthquake SFERN_SOD285 (San Fernando, 2/9/1971, Upland San Antonio Dam, 285 [24]) and the peak acceleration is scaled to $0.35 \mathrm{~m} / \mathrm{s}^{2}$. The NS parameters are set as $\alpha_{1}=0.2$ and $R_{1 y}=6$. The displacement history and acceleration of the $1^{\text {st }}$ floor for the linear structure and the MNS1 are compared in Fig. 5.

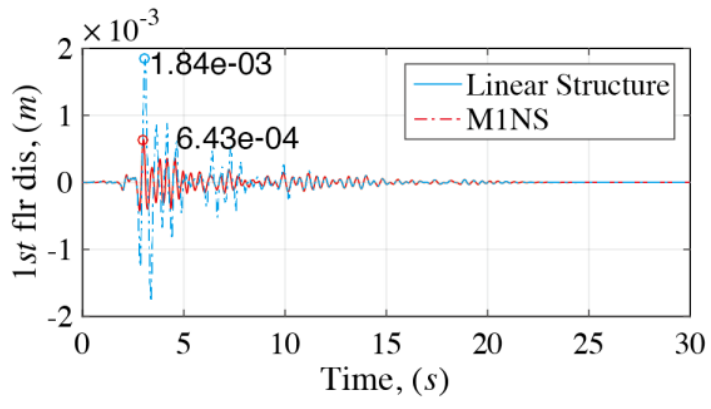

(a)

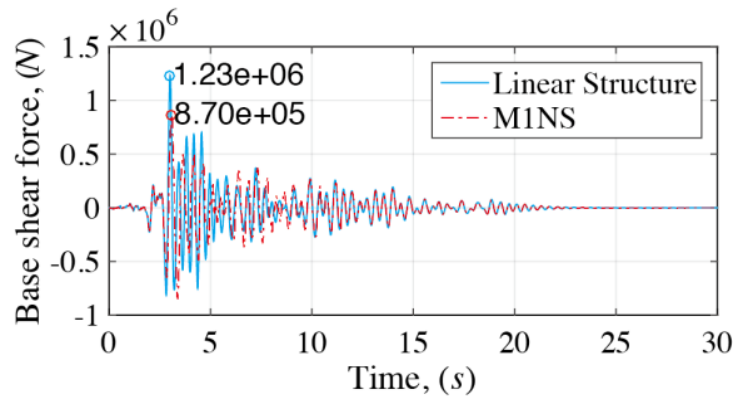

(b)

Fig. 5. Response comparisons for the linear structure and MNS1: (a) displacement; (b) base shear;

In Fig. 5, the solid line is the response of the linear structure, and the dashed line denotes the response of the MNS1. According to the displacement comparison in Fig. 5(a), the MNS1 increases the displacement of linear structure by $85.90 \%$. From the base shear comparison in Fig. 5(b), the MNS1 has the smaller peak value than the linear structure, which means that the NS property reduces the base shear of linear structure by $29.49 \%$.

The seismic excitation can be separated and then imposed on each mode. The separated excitation can be expressed by [13],

$$
-\mathbf{M l} \ddot{\mathbf{x}}_{g}=-\sum_{i=1}^{n} \Gamma_{i} \mathbf{M} \Phi_{i} \ddot{\mathbf{x}}_{g}
$$

The time history response comparisons between the linear structure and MNS1 in the first three 
modes are presented in Fig. 6,
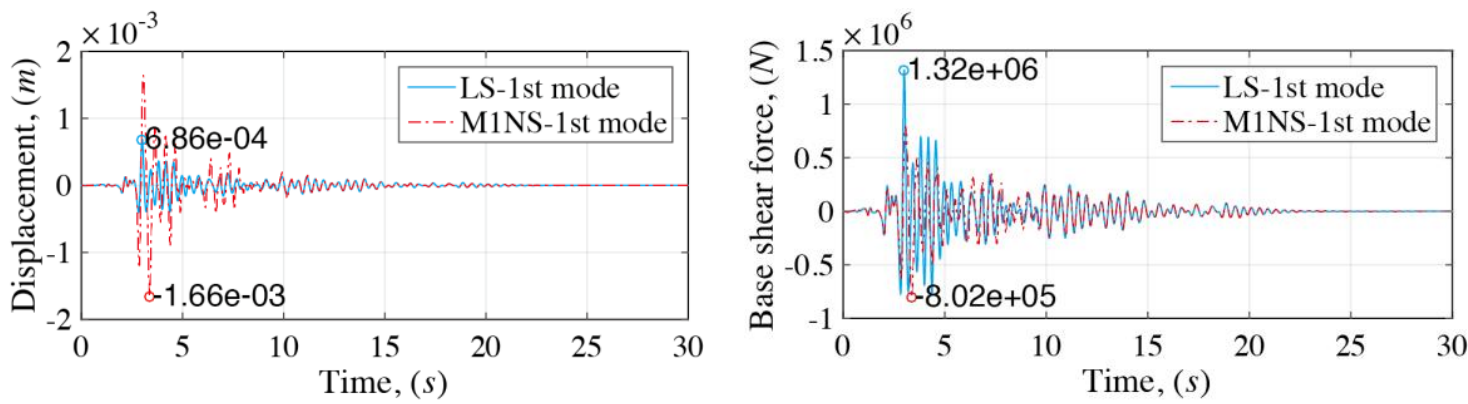

(a)
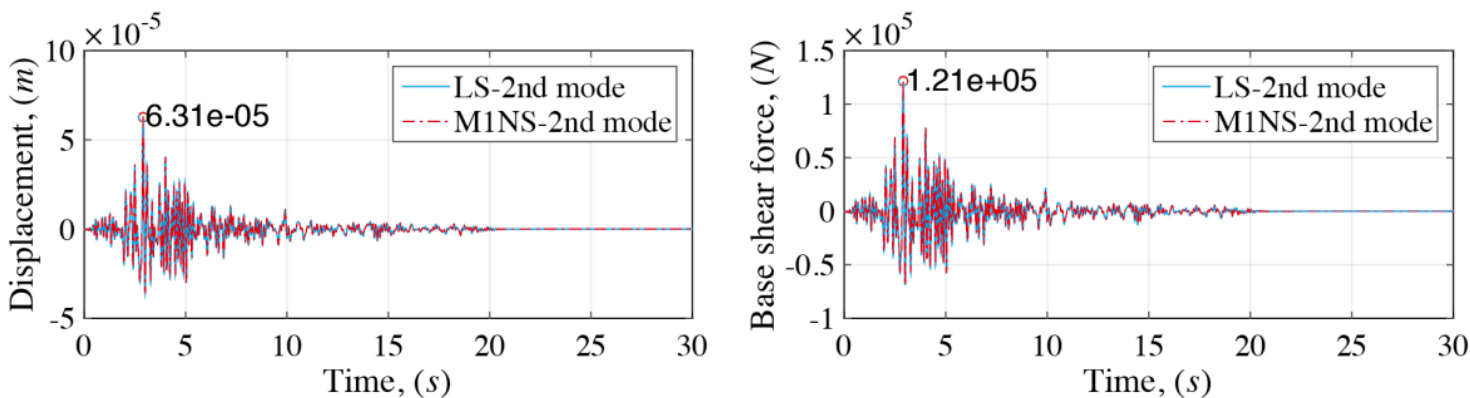

(b)
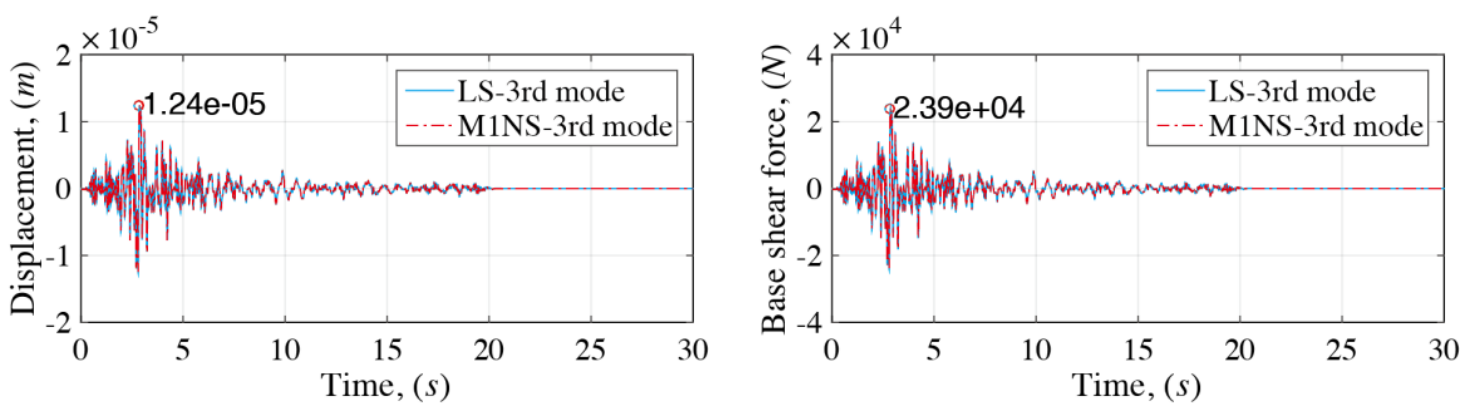

(c)

Fig. 6. Response comparisons for the linear structure and MNS1 under first three modes: (a) $1^{\text {st }}$ mode; (b) $2^{\text {nd }}$ mode; (c) $3^{\text {rd }}$ mode.

In Fig. 6, the LS is the abbreviation of the linear structure. It can be found that the MNS1 on the $2^{\text {nd }}$ mode and $3^{\text {rd }}$ mode has the same response as the linear structure. Because when $R_{1 y}=6$, the "yielding" displacement on the $1^{\text {st }}$ floor is $1.07 \times 10^{-4} \mathrm{~m}$, which is only exceeded by the $1^{\text {st }}$ mode displacement.

\subsection{Accuracy illustration}

The MNS1 response can be estimated by the ASBS in Eq.(19). Due to the approximate equations Eqs.(37) (39), the ASBS would lead to some errors in the response of the MNS1. This section will analyze the accuracy. The $1^{\text {st }}$ floor displacement and base shear are considered, which can be 
estimated by Eqs.(41) (43) when $j=1$.

The displacement and base shear of the MNS1 in Fig. 6 can be compared with the responses estimated by the ASBS or Eq.(41) and Eq.(42) as shown in Fig. 7 and Fig. 8.
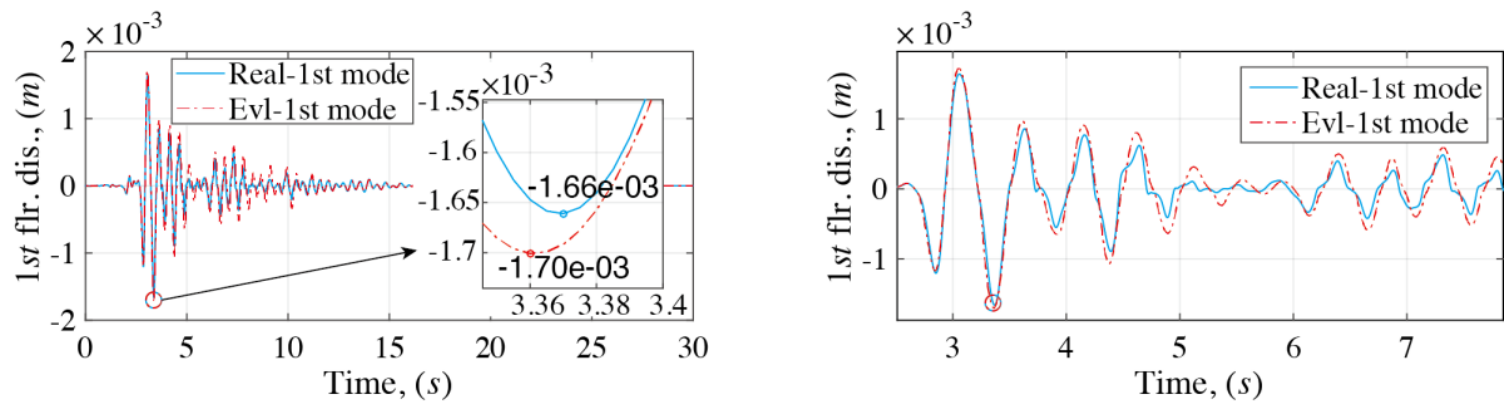

(a)
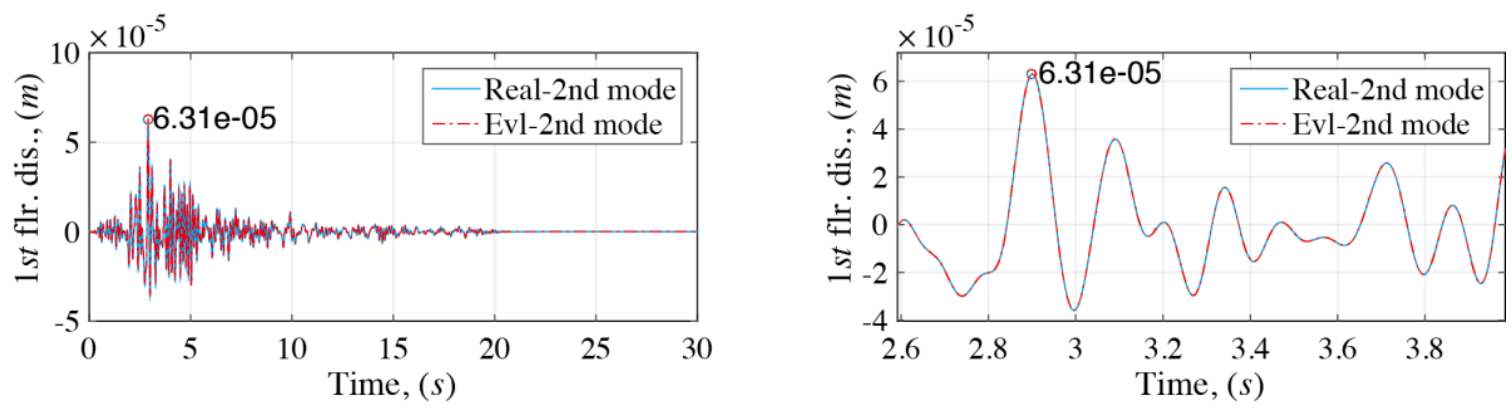

(b)
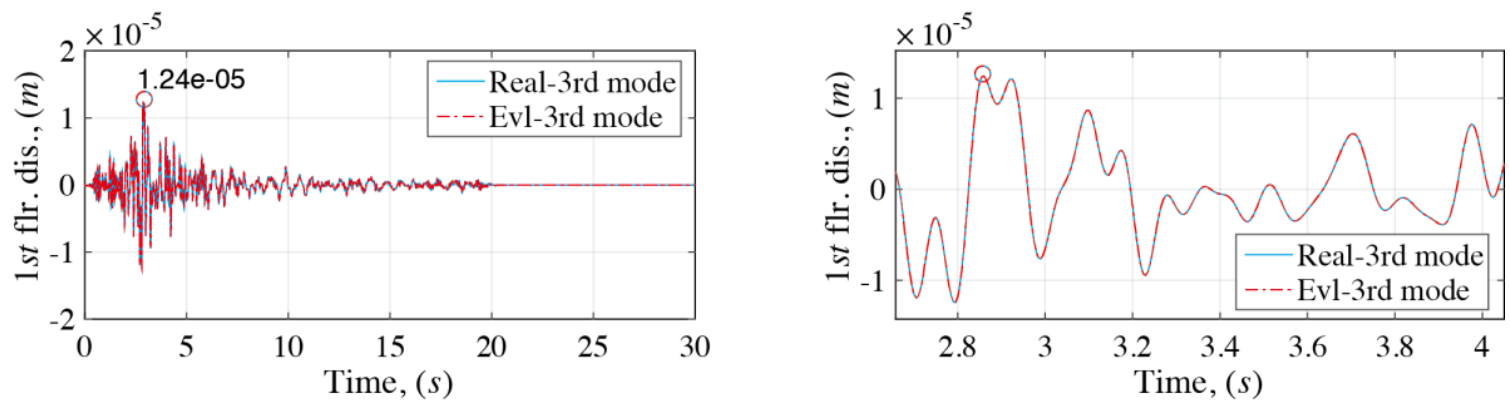

(c)

Fig. 7. Real and estimated displacement comparisons for MNS1 under first three modes: (a) $1^{\text {st }}$ mode; (b) $2^{\text {nd }}$ mode; (c) $3^{\text {rd }}$ mode.

In Fig. 7(a) (c), the solid line represents the MNS1 real response and is denoted as the "Real"; the dashed line is the estimated response by the ASBS and is named as the "Evl". And the right figure shows a zoomed version of the right one. From these figures, approximate error in the $1^{\text {st }}$ mode in Fig. 7(a) is $2.41 \%$. For the comparisons in Fig. 7(b) and Fig. 7(c), there is no error because the displacements in the second and third mode do not cause the "yielding". Through the calculation, the maximal co-ordinate values $\tilde{q}_{i}$ in Eq.(41) for the first three modes are $3.1 \times 10^{-3} \mathrm{~m}, 2.9006 \times 10^{-4} \mathrm{~m}$ and 
$6.8922 \times 10^{-5} \mathrm{~m}$, respectively. The maximal values of the "yielding" co-ordinates $q_{i y}$ for the first three modes are $4.59 \times 10^{-3} \mathrm{~m}, 4.92 \times 10^{-3} \mathrm{~m}$ and $6.68 \times 10^{-3} \mathrm{~m}$, respectively. It is also found that the maximal co-ordinate value $\tilde{q}_{i}$ is smaller than the "yielding" co-ordinate $q_{i y}(i=2,3)$ for the second and third modes. Therefore, there is no error Fig. 7(b) and Fig. 7(c).From the right figures, it is evident that the real and estimated displacement time histories match satisfactorily. Therefore, it can be concluded that the estimated displacements closely approximate the MNS1 real displacements for the first three modes.
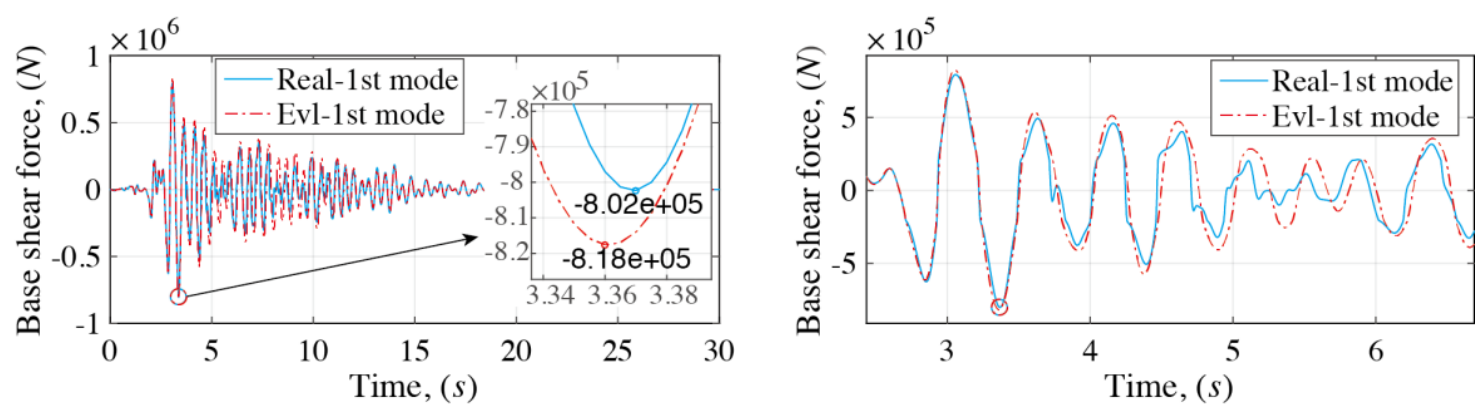

(a)
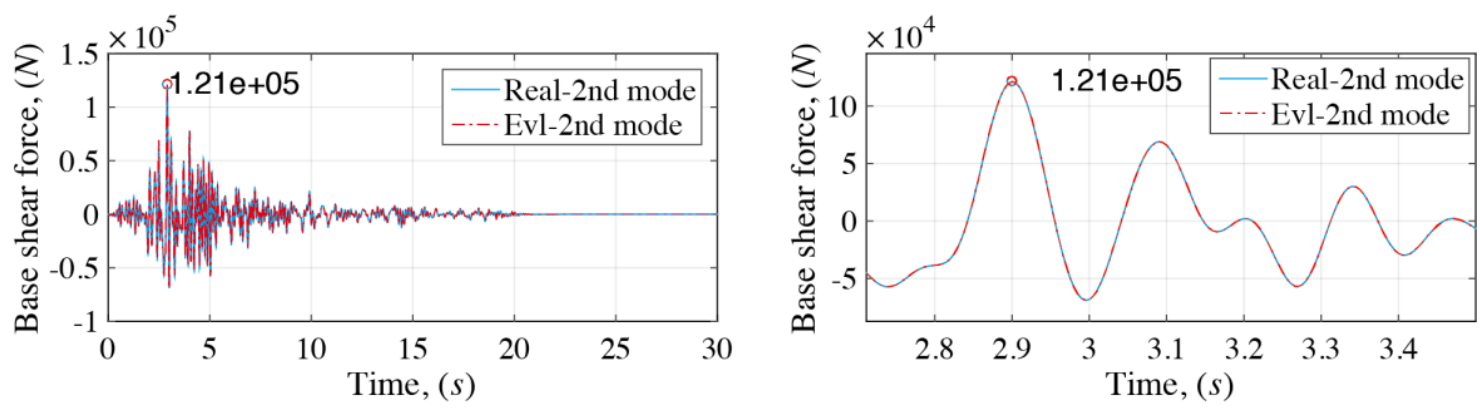

(b)
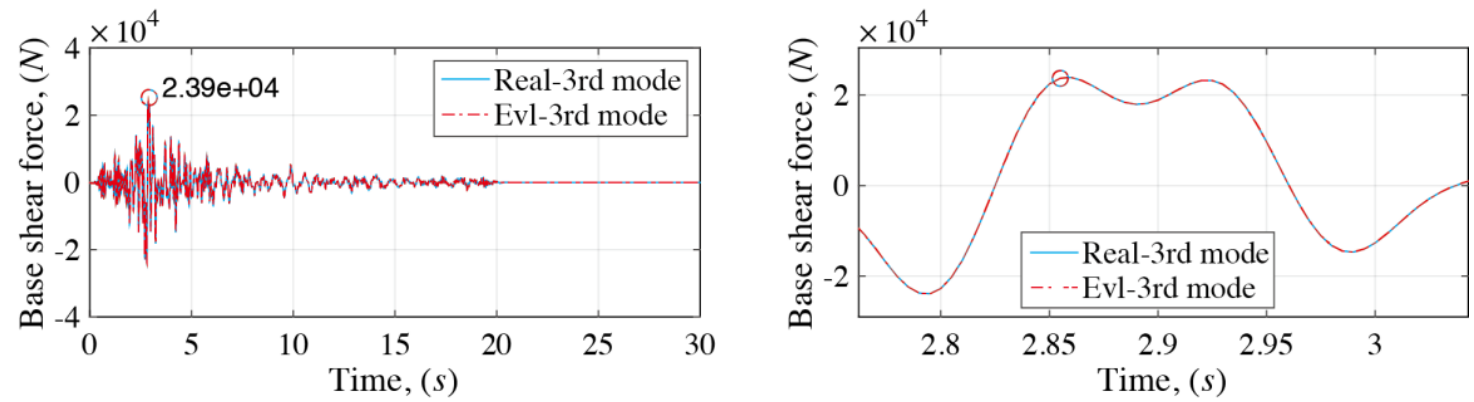

(c)

Fig. 8. Real and estimated base shear comparisons for MNS1 under first three modes: (a) $1^{\text {st }}$ mode; (b) $2^{\text {nd }}$ mode; (c) $3^{\text {rd }}$ mode.

Fig. 8 compares the base shears between the real MNS1 response and the estimated MNS1 response under the first three modes. As described in Fig. 7, the error in approximation in Fig. 8(a) in the $1^{\text {st }}$ mode is $2.00 \%$. There is no error in Fig. $8(\mathrm{~b})$ and Fig. $8(\mathrm{c})$, which has the same reason to that in 
Fig. 7. From the right figures, it demonstrates that the real and estimated base shear time histories match satisfactorily. So the base shear can be estimated satisfactorily by the ASBS in Eq.(19). According to the mode superposition method [12], the estimated displacement in the $1^{\text {st }}$ floor and the base shear can be summed by $\tilde{x}_{1 i}$ in Eq. (41) and $\tilde{V}$ in Eq. (42), respectively. The combined displacement and base shear for the first three modes are compared with the real response in Fig. 9.

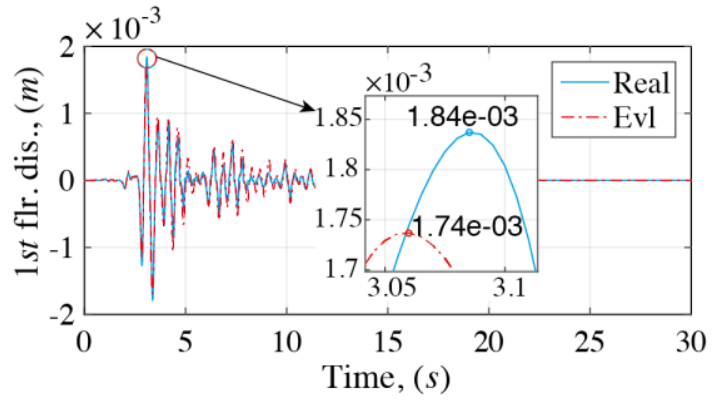

(a)

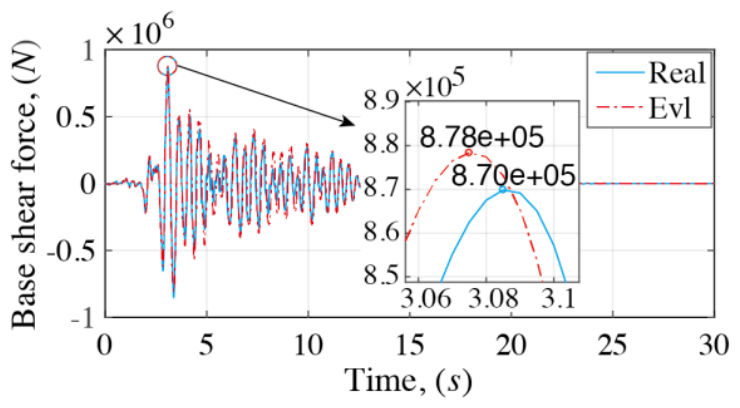

(b)

Fig. 9. Comparisons of real and estimated displacement on $1^{\text {st }}$ floor and base shear

In Fig. 9, the errors of peak values for the $1^{\text {st }}$ floor displacement and the base shear are $5.43 \%$ and $0.92 \%$, respectively. Errors from the evaluation by Eq.(19) are due to the approximation Eqs.(37) (39). In this example, the approximation by Eq.(37) is listed in Table 1. The bold number is the approximate value and is close to 1, which means that the approximation in Eq.(37) is reasonable.

Table 1

Summary of approximation in Eq.(37).

\begin{tabular}{ccccccccc}
\hline$i$ & $\frac{\boldsymbol{\Phi}_{i}^{\mathrm{T}} \mathbf{M} \Psi_{1}}{M_{i}}$ & $\frac{\boldsymbol{\Phi}_{i}^{\mathrm{T}} \mathbf{M} \boldsymbol{\Psi}_{2}}{M_{i}}$ & $\frac{\boldsymbol{\Phi}_{i}^{\mathrm{T}} \mathbf{M} \boldsymbol{\Psi}_{3}}{M_{i}}$ & $\frac{\boldsymbol{\Phi}_{i}^{\mathrm{T}} \mathbf{M} \boldsymbol{\Psi}_{4}}{M_{i}}$ & $\frac{\boldsymbol{\Phi}_{i}^{\mathrm{T}} \mathbf{M} \boldsymbol{\Psi}_{5}}{M_{i}}$ & $\frac{\boldsymbol{\Phi}_{i}^{\mathrm{T}} \mathbf{M} \boldsymbol{\Psi}_{6}}{M_{i}}$ & $\frac{\boldsymbol{\Phi}_{i}^{\mathrm{T}} \mathbf{M} \boldsymbol{\Psi}_{7}}{M_{i}}$ & $\frac{\boldsymbol{\Phi}_{i}^{\mathrm{T}} \mathbf{M} \boldsymbol{\Psi}_{8}}{M_{i}}$ \\
\hline 1 & $\mathbf{0 . 9 7 7 6}$ & -0.2002 & -0.0578 & 0.0258 & -0.0138 & 0.0079 & 0.0044 & -0.0020 \\
2 & 0.1700 & $\mathbf{0 . 9 3 2 9}$ & -0.2984 & 0.0941 & -0.0455 & 0.0250 & 0.0137 & -0.0062 \\
3 & 0.0937 & 0.2398 & $\mathbf{0 . 9 1 4 8}$ & 0.2907 & -0.0968 & 0.0474 & 0.0248 & -0.0109 \\
\hline
\end{tabular}

In order to investigate the suitability of the proposed method for the various site classification, there are four ground motions for four different site classifications to be applied according to Chinese Seismic code [25]. The information of selected earthquakes is listed in Table 2.

Table 2

Summary of selected 4 earthquakes

\begin{tabular}{cccc}
\hline Notation & Name & Information & PGA $(\mathrm{g})$ \\
\hline EqI & Qianan, & Tangshan, Station MO303, 8/9/1976 & 0.1410 \\
\hline
\end{tabular}




\begin{tabular}{cccc}
\hline EqII & El-Centro & Imperial Valley, Station 952, 10/15/1979 & 0.3417 \\
EqIII & AZAK & ChalfantValley,CDMGStation54428, 07/21/1986 & 0.4426 \\
EqIV & ATS & Kocaeli, KOERI, 08/17/1999 & 0.2462 \\
\hline
\end{tabular}

As in Fig. 9, Fig. 10 compares the real and estimated responses under four earthquake records with scaling the PGA to $0.35 \mathrm{~m} / \mathrm{s}^{2}$. From the errors of peak value, it can be found that the displacements and base shears under these earthquakes can be estimated satisfactorily.
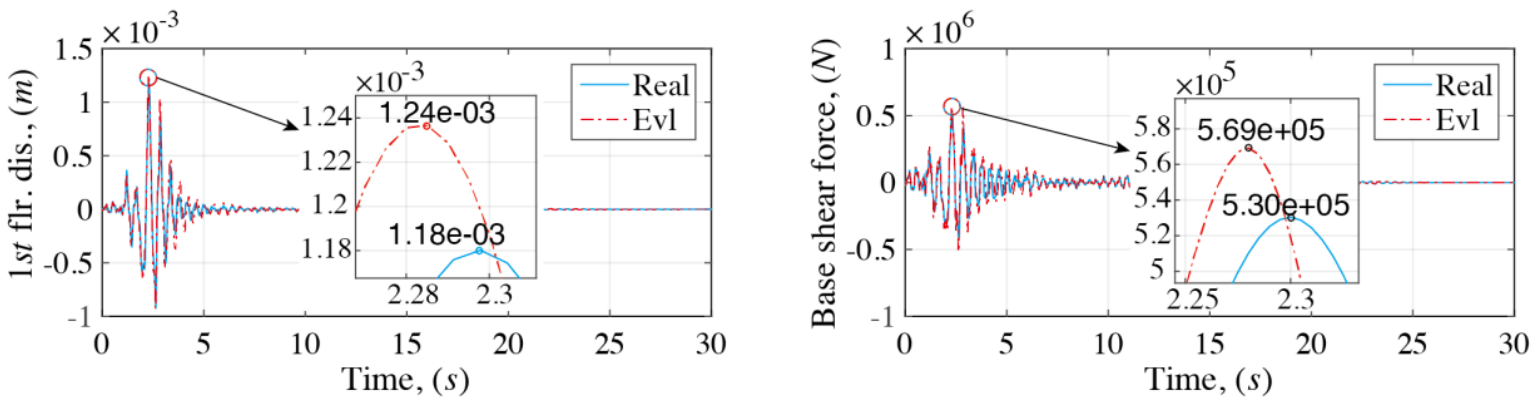

(a)
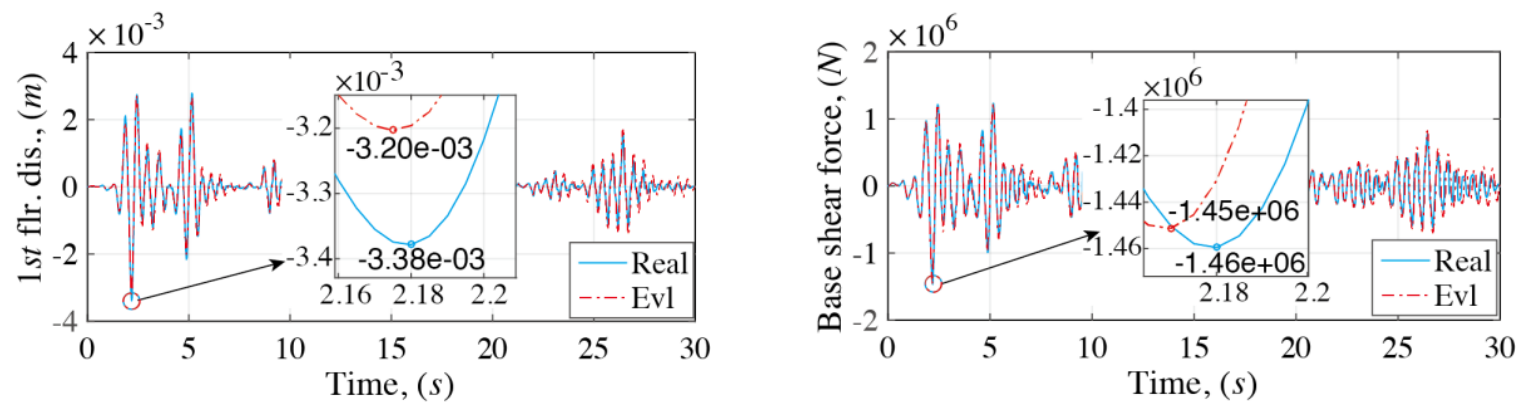

(b)
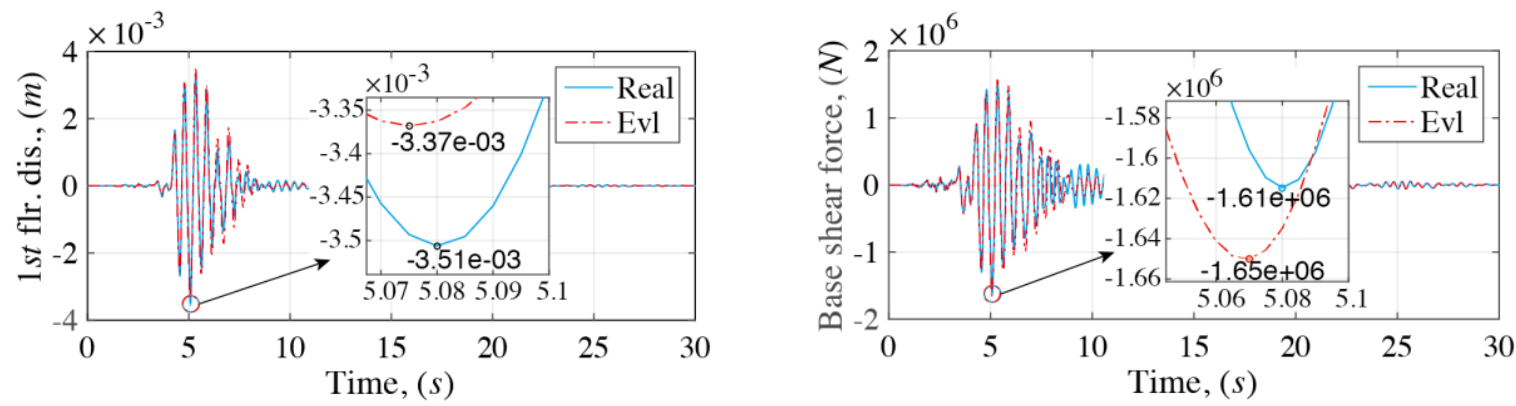

(c) 

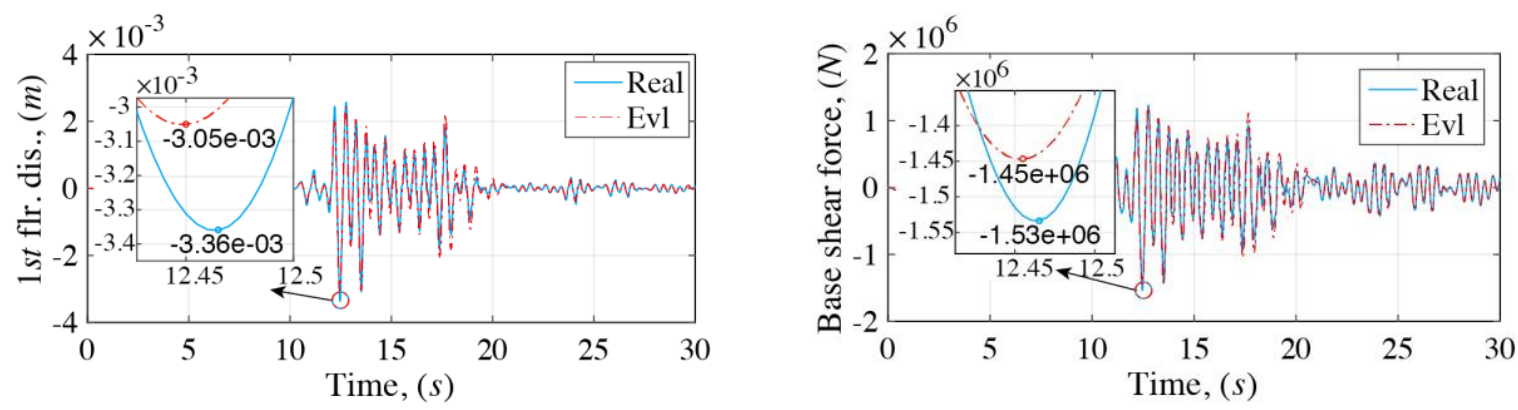

(d)

Fig. 10. Comparisons of real and estimated displacement on $1^{\text {st }}$ floor and base shear: (a) EqI; (b) EqII; (c)EqIII; (d)EqIV

For different NS factors, the "yielding" strength reduction factors $R_{1 y}$ and $\alpha_{1}$, the comparisons of real and estimated responses are also considered. Due to the space limitation, the results are summarized in Table 3. The data in Table 3 shows the errors of the $1^{\text {st }}$ floor displacement peak value and base shear peak value. Taking the first data for example, $\frac{5.08}{7.36}$ means that the error of peak value for the real and estimated displacements on the $1^{\text {st }}$ floor is $5.08 \%$ with the NS factors $\alpha_{1}=0.2$ and $R_{1 y}$ $=6$ under the earthquake EqI; and the error for the base shear is $7.36 \%$.

Table 3

Errors of the peak value for the $1^{\text {st }}$ floor displacement and the base shear under various NS factors and different site classifications

\begin{tabular}{ccccccccccc}
\hline & \multicolumn{3}{c}{$\alpha_{1}=0.2$} & \multicolumn{3}{c}{$\alpha_{1}=0.4$} & \multicolumn{3}{c}{$\alpha_{1}=0.6$} \\
\cline { 2 - 10 } & $R_{1 v}=6$ & $R_{1 y}=4$ & $R_{1 y}=2$ & $R_{1 y}=6$ & $R_{1 v}=4$ & $R_{1 y}=2$ & $R_{1 y}=6$ & $R_{1 y}=4$ & $R_{1 y}=2$ \\
\hline \multirow{2}{*}{ EqI } & $\underline{5.08}$ & 3.01 & 4.10 & 4.53 & 8.03 & 5.04 & 4.02 & 4.01 & 2.88 \\
& 7.36 & 4.02 & 5.06 & 4.59 & 7.32 & 4.05 & 3.95 & 3.74 & 2.53 \\
\hline \multirow{2}{*}{ EqII } & 5.33 & 0.47 & 0.28 & 4.22 & 0.72 & 5.62 & 2.64 & 3.33 & 3.82 \\
& 0.68 & 3.18 & 4.61 & 3.93 & 0.37 & 4.74 & 2.55 & 3.05 & 2.94 \\
\hline \multirow{2}{*}{ EqIII } & 3.99 & 4.82 & 3.97 & 1.40 & 1.33 & 0.39 & 0.73 & 0.34 & 0.85 \\
& 2.48 & 1.25 & 5.85 & 2.19 & 1.70 & 1.68 & 0.68 & 0.30 & 0.25 \\
\hline \multirow{2}{*}{ EqIV } & 9.22 & 5.88 & 1.57 & 1.11 & 0.55 & 0.22 & 1.85 & 1.24 & 0.47 \\
& 5.23 & 3.54 & 0.22 & 1.65 & 1.13 & 0.34 & 1.80 & 1.23 & 0.37 \\
\hline
\end{tabular}

In Table 3, the errors are in a small value region, which means the evaluation by the proposed method is reasonably accurate. In order to explain the applicability for other structures with different natural periods, two kinds of structures are selected by changing the stiffness of the eight-story structure. The stiffness of two structures are 4 times and one fourth of the original structural stiffness, respectively, that is to say, the stiffness are $16 \times 4.8 \times 10^{8} \mathrm{~N} \cdot \mathrm{m}^{2}$ and $4.8 \times 10^{8} \mathrm{~N} \cdot \mathrm{m}^{2}$, respectively. With the 
similar procedure to the original structure, the errors with various NS factors and site classifications for these two structures are presented in Table 4 and Table 5 , respectively.

\section{Table 4}

Errors of the peak value for the structure with the stiffness value equal to $16 \times 4.8 \times 10^{8} \mathrm{~N} \cdot \mathrm{m}^{2}$

\begin{tabular}{ccccccccccc}
\hline & \multicolumn{3}{c}{$\alpha_{1}=0.2$} & \multicolumn{3}{c}{$\alpha_{1}=0.4$} & \multicolumn{3}{c}{$\alpha_{1}=0.6$} \\
\cline { 2 - 10 } & $R_{1 v}=6$ & $R_{1 v}=4$ & $R_{1 v}=2$ & $R_{1 v}=6$ & $R_{1 v}=4$ & $R_{1 v}=2$ & $R_{1 v}=6$ & $R_{1 v}=4$ & $R_{1 v}=2$ \\
\hline \multirow{2}{*}{ EqI } & 5.28 & 14.81 & 0.49 & 2.34 & 2.37 & 1.10 & 0.96 & 1.11 & 1.01 \\
& 4.29 & 12.52 & 0.08 & 2.05 & 1.70 & 1.61 & 0.90 & 1.16 & 1.07 \\
\hline \multirow{2}{*}{ EqII } & 8.79 & 7.78 & 3.31 & 1.11 & 3.23 & 7.83 & 2.02 & 3.38 & 5.63 \\
& 5.93 & 8.34 & 2.72 & 1.47 & 2.84 & 6.17 & 1.90 & 3.07 & 4.67 \\
\hline \multirow{2}{*}{ EqIII } & 4.36 & 1.98 & 13.90 & 1.99 & 2.48 & 1.76 & 1.26 & 0.57 & 1.65 \\
& 3.60 & 1.32 & 7.46 & 1.73 & 2.76 & 0.69 & 1.06 & 0.31 & 1.87 \\
\hline \multirow{2}{*}{ EqIV } & 6.89 & 2.84 & 9.03 & 0.96 & 0.54 & 4.16 & 1.82 & 1.20 & 2.52 \\
& 6.16 & 2.23 & 3.63 & 0.77 & 0.42 & 2.43 & 1.56 & 0.86 & 1.80 \\
\hline
\end{tabular}

Table 5

Errors of the peak value for the structure with the stiffness value equal to $4.8 \times 10^{8} \mathrm{~N} \cdot \mathrm{m}^{2}$

\begin{tabular}{ccccccccccc}
\hline & \multicolumn{3}{c}{$\alpha_{1}=0.2$} & \multicolumn{3}{c}{$\alpha_{1}=0.4$} & \multicolumn{3}{c}{$\alpha_{1}=0.6$} \\
\cline { 2 - 10 } & $R_{1 y}=6$ & $R_{1 y}=4$ & $R_{1 y}=2$ & $R_{1 y}=6$ & $R_{1 y}=4$ & $R_{1 y}=2$ & $R_{1 y}=6$ & $R_{1 y}=4$ & $R_{1 y}=2$ \\
\hline \multirow{2}{*}{ EqI } & 10.02 & $\mathbf{1 5 . 3 0}$ & 7.74 & 4.50 & 3.39 & 1.45 & 0.14 & 0.11 & 0.27 \\
& 0.02 & 8.03 & 14.71 & 5.00 & 9.71 & 11.40 & 6.15 & 7.88 & 7.50 \\
\hline \multirow{2}{*}{ EqII } & 0.48 & 3.65 & 10.26 & 2.08 & 3.47 & 9.46 & 0.31 & 1.19 & 2.16 \\
& 0.27 & 8.84 & 8.26 & 1.58 & 1.52 & 5.87 & 0.47 & 0.79 & 1.08 \\
\hline \multirow{2}{*}{ EqIII } & 8.49 & 8.49 & 1.79 & 0.78 & 0.68 & 0.57 & 1.27 & 1.04 & 0.06 \\
& 2.46 & 3.90 & 0.92 & 4.24 & 2.91 & 0.87 & 2.67 & 2.09 & 0.53 \\
\hline \multirow{2}{*}{ EqIV } & 1.27 & 2.61 & 4.50 & 1.58 & 1.85 & 12.82 & 5.87 & 7.84 & 6.98 \\
& 3.25 & 3.23 & 2.06 & 0.65 & 2.07 & 4.98 & 8.26 & 6.26 & 1.60 \\
\hline
\end{tabular}

Most of the errors in Table 4 and Table 5 are small enough, except for a few errors around 10\%. Take the error $=15.30 \%$ for example in Table 5 . The structural stiffness is one fourth of the original stiffness, and with the NS factors $\alpha_{1}=0.2$ and $R_{1 y}=4$ under the earthquake EqI. Fig. 11 shows the displacement comparison for this case. It can be observed that the estimated displacement is very close to the real displacement in all time series. To describe the accuracy, a correlation coefficient $\mathrm{r}_{\text {Real,Evl }}$ is defined in Eq.(45).

$$
r_{R e a l, E v l}=\frac{\mathrm{E}((\text { Real }-\mathrm{E}(\text { Real }))(E v l-\mathrm{E}(E v l)))}{\sqrt{\operatorname{Var}(\text { Real })(\operatorname{Var}(E v l)}}
$$


where $\mathrm{E}(\cdot)$ denotes the average value, $\operatorname{Var}(\cdot)$ represents the variance value, and Real and $E v l$ are the time histories of the real displacement and estimated displacement, respectively. The correlation coefficient $\mathrm{r}_{\text {Real,Evl }}=93.5 \%$, which illustrates how close the estimated displacement is with the real displacement.

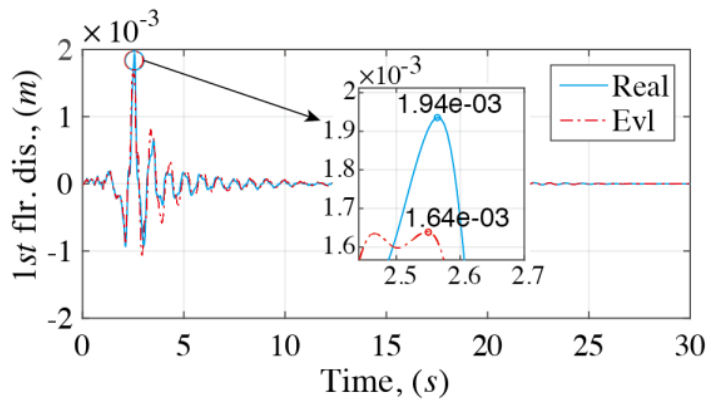

(a)

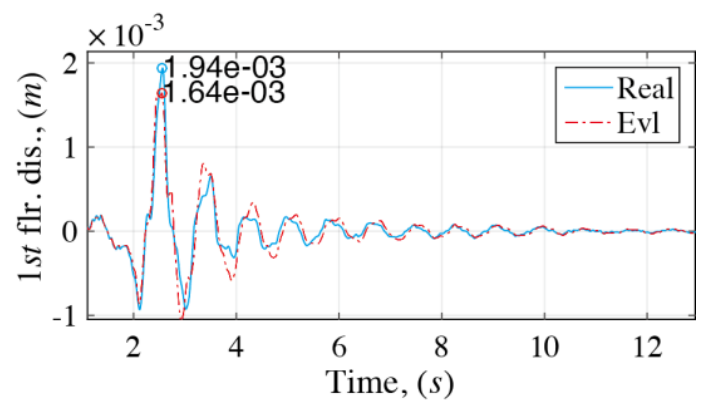

(b)

Fig. 11. Comparisons of real and estimated displacement on $1^{\text {st }}$ floor with NS factors $\alpha_{1}=0.2$ and $R_{1 y}$ $=4$ under earthquake EqI;

Although there are some errors around 10\%, the real and estimated displacement time histories match each other satisfactorily. On the other hand, almost all of the errors are small enough. Therefore, this evaluation is acceptable. It can be concluded that the proposed method is capable of evaluating the response of the MNS1.

It should be noted that although all of the numerical examples are building structures, the proposed method is also suitable for the applications for other particular examples that satisfies Eq.(1) and Fig. 2.

\section{Conclusions}

This paper aims to develop a new method using the single degree of freedom system (SDOF) to estimate the response of the multi degree of freedom (MDOF) structure with the negative stiffness (NS) in the $1^{\text {st }}$ story (MNS1), which is a fundamental research for building a new spectrum to estimate the seismic demands of a structure with the NS. It has led to the following conclusions:

MNS1 that has the bilinear elastic behavior due to the NS in the $1^{\text {st }}$ story (MNS1) can be approximately represented by the SDOF bilinear elastic system (ASBS). As the mode superposition for the linear elastic MDOF structure [12], the MNS1 can also be decomposed along with its modes, where there exists the coupling phenomenon in the co-ordinates of this decomposed system. ASBS is deduced by neglecting the coupling, whose "yielding" co-ordinate is the "yielding" deformation in the $1^{\text {st }}$ story of MNS1 divided by the value of the corresponding mode shape in the $1^{\text {st }}$ story placement as described in Eq.(22). The stiffness of ASBS after "yielding" is the square of the natural frequency for 
the structure with the "yielding" stiffness $\hat{\mathbf{K}}$ in Eq.(10). The accuracy of the approximation obtained by neglecting the coupling is theoretically derived by the sensitivity method of mode shapes that investigates the relative changes in structural mode shapes due to the variation of stiffness. The derivation demonstrates that the value of the neglecting coupling is always much smaller than the one with the coupling, which means that the approximation coupling is reasonable.

An eight-story numerical example is employed to illustrate the accuracy of approximation of the proposed method, where the estimated responses - including the $1^{\text {st }}$ floor displacements and the base shears under the first three modes, the combined $1^{\text {st }}$ floor displacement and the combined base shear under the first three modes - are compared with the real responses that involve the responses under the first three modes and the total responses. The results for the structure with NS factors $\alpha_{1}=0.2$ and $R_{1 y}=6$ under the excitation SFERN_SOD285 with the peak acceleration scaled to $0.35 \mathrm{~m} / \mathrm{s}^{2}$ shows that the approximate procedure provides good estimates of floor displacements and base shears. The responses of the structures with various NS factors under four different site classifications in Chinese Seismic code are also compared, which demonstrate that most estimates are accurate enough. For the cases with the errors around $10 \%$, they have the high values of the correlation coefficients $\mathrm{r}_{\text {Real,Evl }}$ defined in Eq.(45), which indicates that the real and estimated displacement time histories match satisfactorily. Thus, the proposed method seems to estimate the responses of MNS1 accurately enough for practical application in building evaluation and design.

This paper has focused on the evaluation of MNS1, which can help to reduce the structural base shear with the NS in first story. The evaluation errors around 10\% may be caused by the earthquakes under some site classification whose frequency spectrum may need to be considered, and by the NS factors that determine the bilinear properties of the structure. These factors can affect the accuracy of the statement about the analyzed result, i.e. $\left(\alpha_{1}-1\right) k_{1} \phi_{1 i} \phi_{1 j} /\left(\omega_{j}^{2}-\omega_{i}^{2}\right) / M_{i}$ in Eqs. (34) (36) is much smaller than 1. On the other hand, the evaluation of a MDOF structure with NS on different stories should also be analyzed. Work along these lines is in progress.

\section{ACKNOWLEDGEMENT}

The research is supported from National Natural Science Foundation of China (NSFC) under grant number 51261120375 and 51421064 from Prof. Hongnan Li, the NSFC under grant number 51408099 and China Postdoctoral Science Foundation under grant number 2014M560210 from Dr. Chunxu Qu, and National Science Foundation under grant NSF CMMI NEESR 0830391 from Prof. Satish Nagarajaiah.

\section{REFERENCES}

[1] A.G. Tsonos, An innovative solution for strengthening of old R/C structures and for improving the FRP strengthening method, Structural Monitoring and Maintenance 1 (3) (2014) 323-338. 
[2] G.I. Kalogeropoulos, A.G. Tsonos, Effectiveness of R/C jacketing of substandard R/C columns with short lap splices, Structural Monitoring and Maintenance 1 (3) (2014) 273-292.

[3] A.M. Reinhorn, S. Viti, G.P. Cimellaro, Retrofit of structures: Strength reduction with damping enhancement, Proceedings of the 37th UJNR Panel Meeting on Wind and Seismic Effects, Public Works Research Institute Tsukuba-shi, Japan, 2005.

[4] S. Viti, G.P. Cimellaro, A.M. Reinhorn, Retrofit of a hospital through strength reduction and enhanced damping, Smart Structures and Systems 2 (4) (2006) 339-355.

[5] X. Huang, Y. Chen, H. Hua, X. Liu, Z. Zhang, Shock isolation performance of a nonlinear isolator using Euler buckled beam as negative stiffness corrector: Theoretical and experimental study, Journal of Sound and Vibration 345 (2015) 178-196.

[6] W. Wu, X. Chen, Y. Shan, Analysis and experiment of a vibration isolator using a novel magnetic spring with negative stiffness, Journal of Sound and Vibration 333 (13) (2014) 2958-2970.

[7] I. Antoniadis, D. Chronopoulos, V. Spitas, D. Koulocheris, Hyper-damping properties of a stiff and stable linear oscillator with a negative stiffness element, Journal of Sound and Vibration 346 (2015) 37-52.

[8] S. Nagarajaiah, A.M. Reinhorn, M.C. Constantinou, D. Taylor, D.T.R. Pasala, A.A. Sarlis, True adaptive negative stiffness: A new structural modification approach for seismic protection, Proceedings of the 5th World Conf. on Structural Control and Monitoring, International Association of Structural Control and Monitoring, Univ. of Tokyo, Japan, 2010.

[9] A.A. Sarlis, D.T.R. Pasala, M.C. Constantinou, A.M. Reinhorn, S. Nagarajaiah, D. Taylor, Negative stiffness device for seismic protection of structures, Journal of Structural Engineering, ASCE 139 (7) (2013) 1124-1133.

[10] A.A. Sarlis, D.T.R. Pasala, M.C. Constantinou, A.M. Reinhorn, S. Nagarajaiah, D.P. Taylor, Negative Stiffness Device for Seismic Protection of Structures, Technical Report MCEER-13-0005, Multidisciplinary Center for Earthquake Engineering Research, State University of New York at Buffalo, Buffalo, NY, 2013.

[11] D.T.R. Pasala, A.A. Sarlis, S. Nagarajaiah, A.M. Reinhorn, M.C. Constantinou, D.P. Taylor, Seismic Response Control of Structures Using a Novel Adaptive Passive Negative Stiffness Device, Technical Report MCEER-13-0004, Multidisciplinary Center for Earthquake Engineering Research, State University of New York at Buffalo, Buffalo, NY, 2013.

[12] A. Chopra, Dynamics of Structures: Theory and Applications to Earthquake Engineering. Prentice-Hall, Englewood Cliffs, New Jersey, U.S.A., 2001.

[13] A.K. Chopra, R.K. Goel, A modal pushover analysis procedure for estimating seismic demands for buildings, Earthquake Engineering \& Structural Dynamics 31 (3) (2002) 561-582.

[14] T. Makarios, Equivalent non-linear single degree of freedom system of spatial asymmetric multi-storey buildings in pushover procedure: theory and applications, Journal of the Structural Design of Tall \& Special Buildings 18 (7) (2009) 729-763.

[15] T. Tjhin, M. Aschheim, E. Hernández-Montes, Estimates of Peak Roof Displacement Using “Equivalent” Single Degree of Freedom Systems, Journal of Structural Engineering, 
ASCE 131 (3) (2005) 517-522.

[16] D. Vamvatsikos, C.A. Cornell, Direct estimation of the seismic demand and capacity of oscillators with multi-linear static pushovers through IDA, Earthquake Engineering \& Structural Dynamics 35 (9) (2006) 1097-1117.

[17] D.T.R. Pasala, A.A. Sarlis, S. Nagarajaiah, A.M. Reinhorn, M.C. Constantinou, D. Taylor, Adaptive negative stiffness: A new structural modification approach for seismic protection, Journal of Structural Engineering, ASCE 139 (2013) 1112-1123.

[18] T. Ray, A.M. Reinhorn, S. Nagarajaiah, Nonlinear elastic and inelastic spectra with inherent and supplemental damping, Earthquake Engineering and Structural Dynamics 42 (14) (2013) 2151-2165.

[19] D.T.R. Pasala, A.A. Sarlis, A.M. Reinhorn, S. Nagarajaiah, M.C. Constantinou, D. Taylor, Simulated bilinear-elastic behavior in a SDOF elastic structure using negative stiffness device: Experimental and analytical study., Journal of Structural Engineering, ASCE 140 (2) (2014) 04013049.

[20] J. Yang, Y.P. Xiong, J.T. Xing, Dynamics and power flow behaviour of a nonlinear vibration isolation system with a negative stiffness mechanism, Journal of sound and vibration 332 (1) (2013) 167-183.

[21] K. Zou, S. Nagarajaiah, Study of a piecewise linear dynamic system with negative and positive stiffness, Communications in Nonlinear Science and Numerical Simulation 22 (1) (2015) 1084-1101.

[22] J. Zhao, J.T. DeWolf, Sensitivity study for vibrational parameters used in damage Journal of Structural Engineering 125 (1999) 410-416.

[23] G. Li, H. Li, Y. Zhang, Displacement estimation of nonlinear structures using the force analogy method, The Structural Design of Tall and Special Buildings 24 (1) (2015) 59-72.

[24] PEER, http://ngawest2.berkeley.edu, 2015.

[25] GB50011-2010: 2010, Code for seismic design of buildings, China architecture and building press, Beijing, China. 